\title{
Nanoparticle Technology: An alternative approach for Leishmaniasis Treatment
}

\author{
Aldo Eloizo Job, Alexandre Fioravante de Siqueira, \\ Caroline Silva Danna, Felipe Silva Bellucci, \\ Flávio Camargo Cabrera, \\ Leandra Ernst Kerche Silva and \\ Pedro Henrique Benites Aoki \\ Additional information is available at the end of the chapter \\ http://dx.doi.org/10.5772/57283
}

\section{Introduction}

Leishmaniasis is an infection caused by a parasite from Leishmania genus, which can manifest itself through different forms: cutaneous (with skin ulcers) [1, 2], visceral (hepato and splenomegaly) [3], and diffuse integumentary form (injuries beyond the superficial skin barrier, hitting cartilages and deeper connective tissues) [4]. Leishmaniasis vectors are diptera from Psychodida family, which includes hematophagous from Phlebotomus genus (Old World) and Lutzomuyia genus (New World), with wide distribution in warm and temperate climates [5] (Figure 1).

In the New World, eight Leishmania species are responsible for men diseases: Leishmania braziliensis, Leishmania guyanensis, Leishmania panamensis, Leishmania lainsoni, Leishmania mexicana, Leishmania amazonensis, Leishmania venezuelensis and Leishmania chagasi [7]. Thus, leishmaniasis can be found in four continents, being considered endemic in 88 countries, of which 72 are in development [5].

Different protozoa species are able to live inside sand flies (insect vectors) from Phlebotominae subfamily and vertebrate hosts. This parasite lives between two different types of hosts due to its significant morphological changes. In vertebrate hosts, Leishmania is at amastigote form without flagella and inside the digestive tract of the sand flies, the parasite is flagellated and fusiform, and receives the name of promastigote, as shown in Figure 2. 


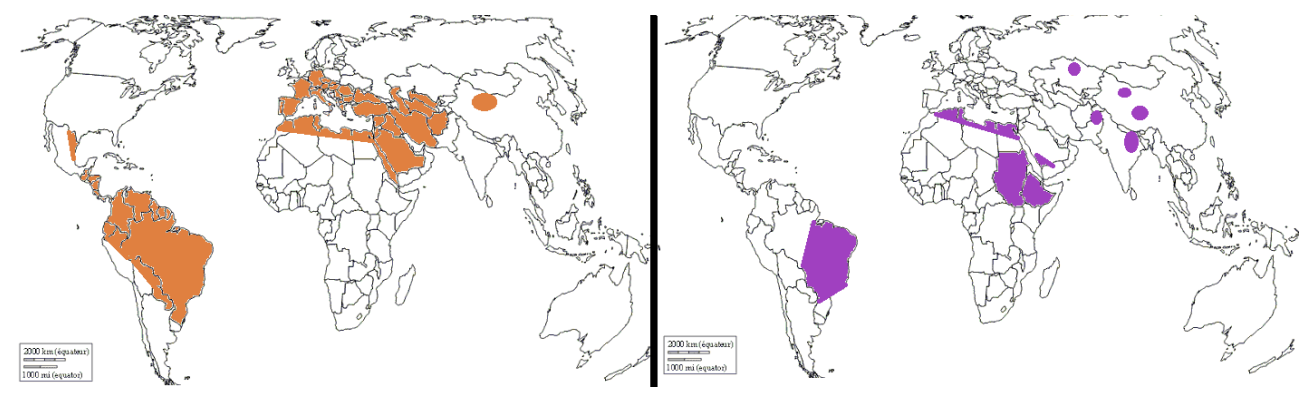

Figure 1. Distribution in Old World and New World of cutaneous (left, marked in orange) and visceral (right, marked in purple) leishmaniasis. Affected areas marked according to the World Health Organization [6].

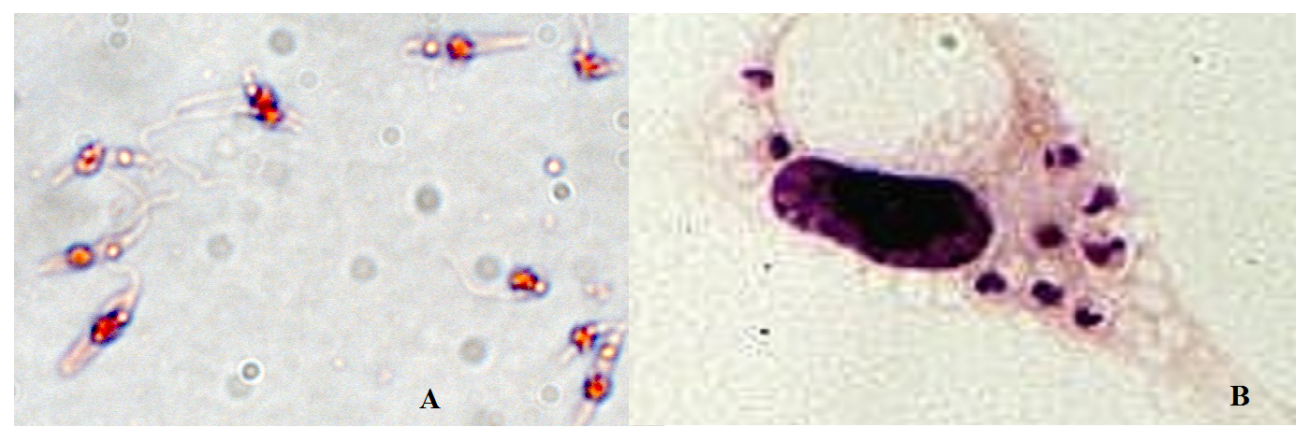

Figure 2. Leishmania sp (A) promastigote and (B) amastigote (right) forms.

In Figure 3, two important cellular structures used to identify the Leishmania parasites are highlited in the first image - the nucleus, indicated by the letter $\mathrm{N}$ and the kinetoplast, indicated by the letter $\mathrm{K}$. These structures are kept in the distinct methacyclogenesis stages. After completing the cycle modifications, Leishmania becomes able to infect mammalian cells.

Different forms of leishmaniasis are transmitted through the bite of female sand flies. The transmission cycle begins when, during a bite, the mosquito ingests mammalian blood infected with amastigote forms. Once installed in the digestive tract of the host, Leishmania passes through the process of differentiation, methacyclogenesis, and the new form of the parasite, promastigote, can be inject in mammalian blood again during a bite, thus completing the disease transmission cycle (Figure 4). 


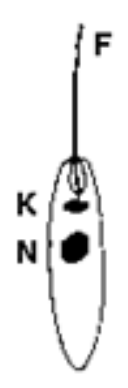

a

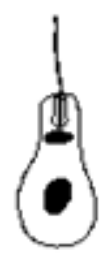

e

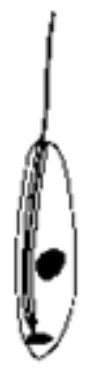

b

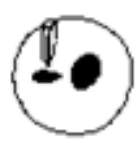

$\mathbf{f}$

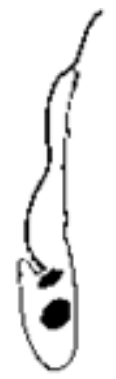

c

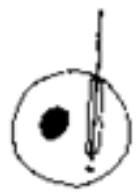

g

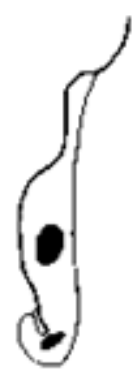

d

Figure 3. Different transition stages between (a) promastigote and (f) amastigote forms of Leishmania braziliensis.

Insect Cycle (1) Phlebotominae sand fly injects promastigotes
into the skin while taking a blood meal

(7)

Promastigotes divide in the midgut of the insect igrate to the proboscis

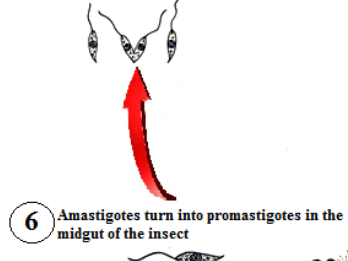

ras
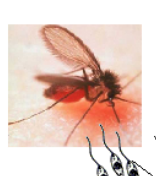

2.
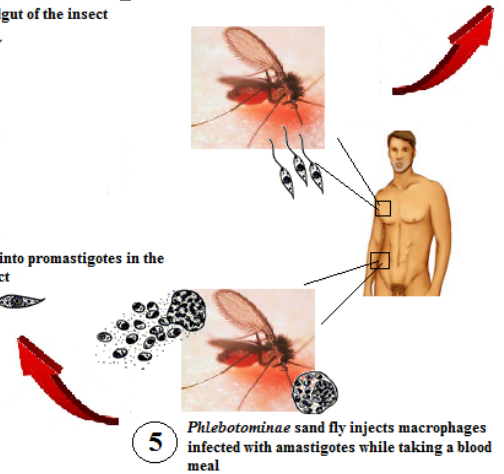

Human Cycle

(2)

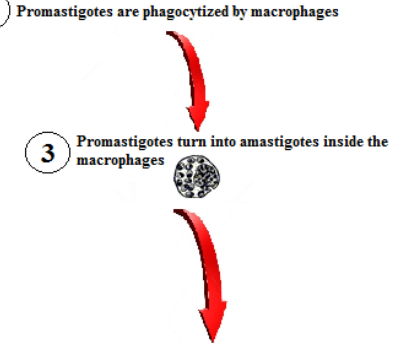

(4) Amastigotes multiply in cells of various tissue

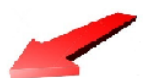

Figure 4. Life cycle of the parasite. 


\section{Current treatments}

In 1912, Vianna [9] observed that the tartar emetic (an antimony compound) was effective in the treatment of American Integumentary Leishmaniasis. Due to toxicity and serious collateral damages associated to the tartar emetic use, i.e. gastrointestinal intolerance and cardiotoxic effects, the trivalent antimonials (Table 1) were replaced by quinquivalent compounds (Table 2). In 1936, Schmidt introduced in medical therapy antimony (V) sodium gluconate, commercially known as Solustibosan ${ }^{\circledR}$ (Bayer) or Pentostam ${ }^{\circledR}$ (Glaxo Wellcome) $[10,11]$.

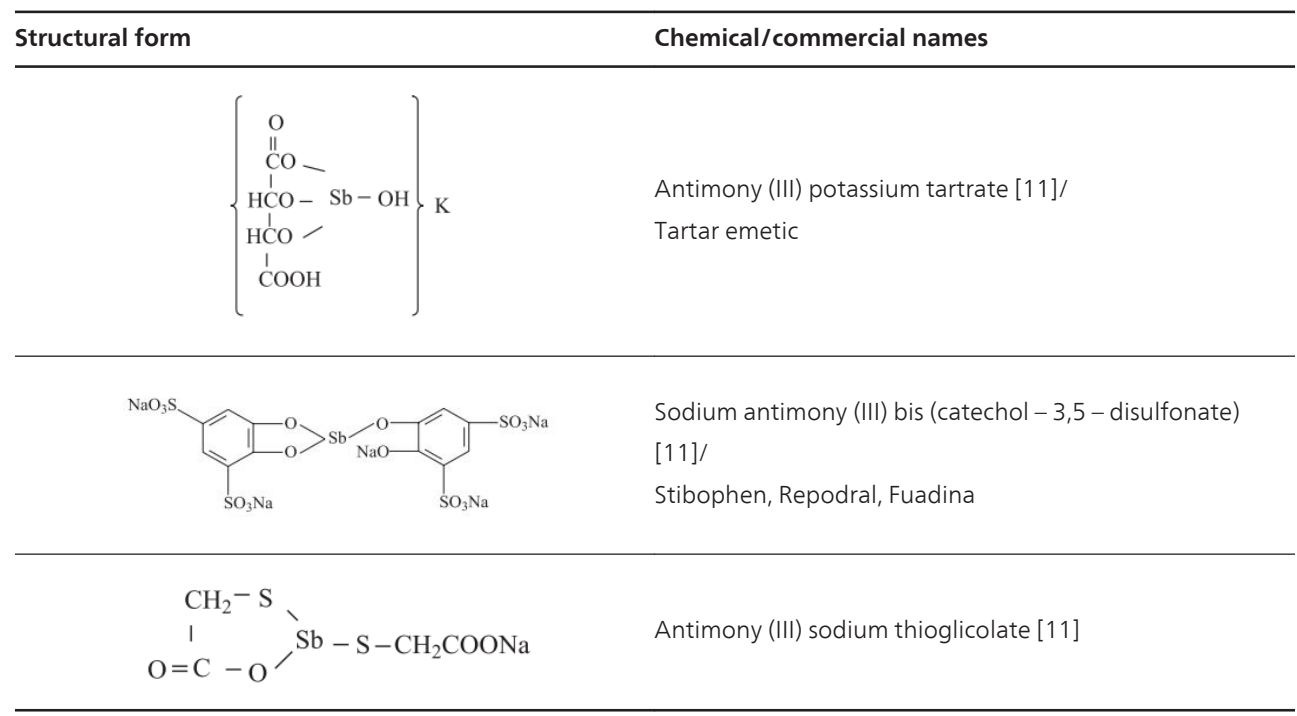

Table 1. Chemical structure of trivalent antimonials used in medical clinic, with their respective chemical and commercial names [5].

One of the most commonly used drugs is N-methylglucamine antimoniate, which are especially effective in the treatment of cutaneous and visceral leishmaniasis. This drug provokes fast regression of the clinical and hematological manifestations of the disease, as well as the dead of the parasite [5]. However, due to its low dosages and discontinuous treatments, some therapy failures, an increase of the resistant forms of the parasites started to show up [12-14].

The World Health Organization preconizes that antimonials dosages Should not trespass 20 $\mathrm{mg} / \mathrm{kg} / \mathrm{day}$, and due to its elevated toxicity, the dosage of antimony ingested per day should not be higher than $850 \mathrm{mg}$ [12]. However, the N-methylglucamine antimoniate is rapidly absorbed and about $90 \%$ of the antimony ingested is excreted through the kidneys in the firsts 48 hours [15]. Consequently, there is a necessity to administrate high doses of the drug, in continuous regimen, so an elevated dosage of antimony is assured in the tissues, and therefore the treatment efficacy is obtained. 


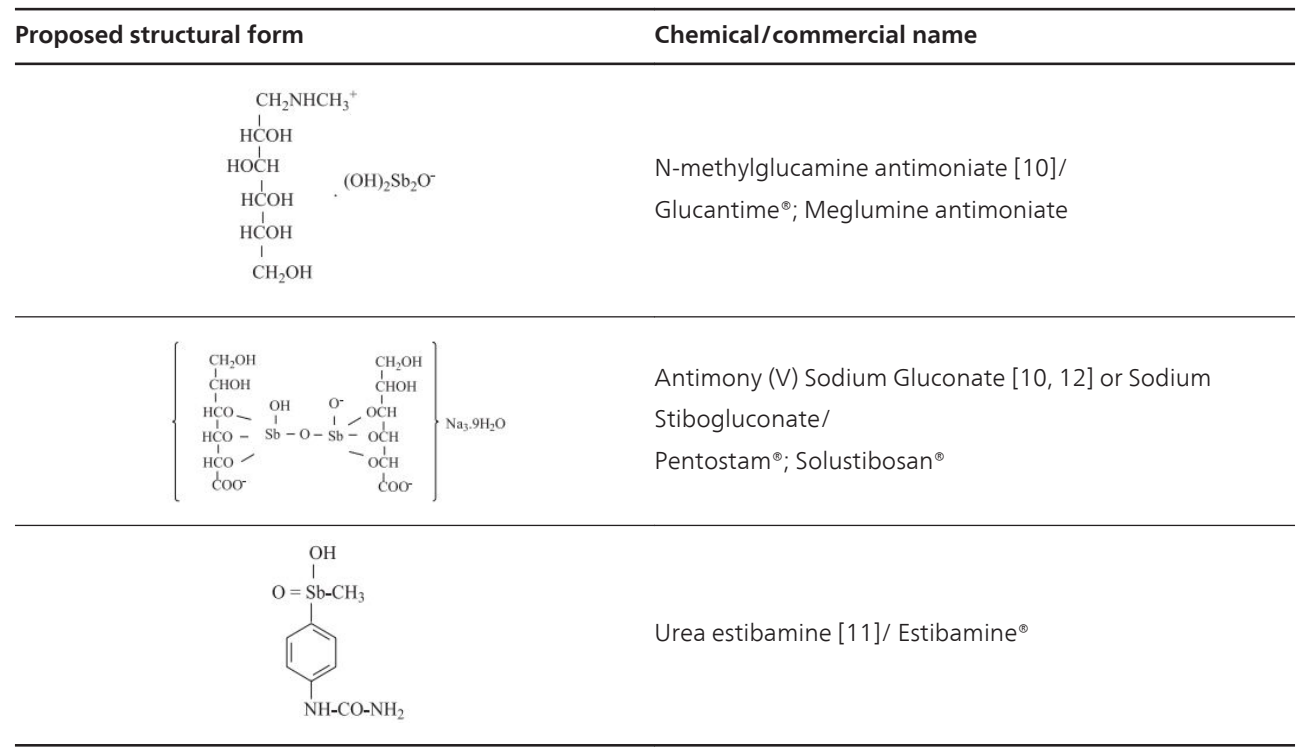

Table 2. Chemical structure of quinquivalent antimonials used in medical clinic [5].

High doses of N-methylglucamine antimoniate generate a various diversity of collateral effects, such as nephritis, gastrointestinal, cardiovascular and respiratory disturbances. In some cases, besides destroying the parasites, some patients are led to death [11]. The antimony can still be detected in the hair of the patients a year after the treatment is finished [16]. The toxicity of antimony can be explained by the fact that there are some evidences describing that a metabolic conversion happens inside the macrophages transforming antimony $\mathrm{V}\left(\mathrm{Sb}^{5+}\right)$ in antimony III $\left(\mathrm{Sb}^{3+}\right)$ [13] and the antimony III is proved to be more lethal to the Leishmania species [5]. So the hypothesis is that the antimony $\mathrm{V}$ works as prodrug, and the conversion to antimony III is what guarantee its efficacy after administration [17], and the antimony III interferes in the $\beta$-oxidation process of fat acids of the parasites, as so in the glycolysis, taking the ATP to a low level inside the cell [12]. This way, the antimony III would be responsible for the toxicity of the drug as well as its therapeutic activity [18].

Besides antimonials, other medicines have been used in the treatment of the various forms of leishmaniasis, among them can be found pentamidine, amphotericin B, paromomycin, and miltefosine (Table 3) [5]. Pentamidine is also effective in the trypanosomiasis treatment [19] and this drug is also highly toxic, showing as collateral effects hypoglycemia, hypotension, cardiologic changes, nephrotoxicity and even death [12]. Amphotericin B is an antibiotic produced by Streptomyces nodosus and can produce nephrotoxicity, depleting the potassium and magnesium levels of the organism [20]. Paromomycin is an aminoglycoside antibiotic active against Leishmania species in in vitro and in vivo forms. It is been highly tested in India, where antimonial standard treatments are not effective [5]. Miltefosine is an alkyl phospholipidic anticancer drug and it is in intensive tests also in India. It is showing excellent results and may be the first oral treatment against visceral leishmaniasis [21]. 


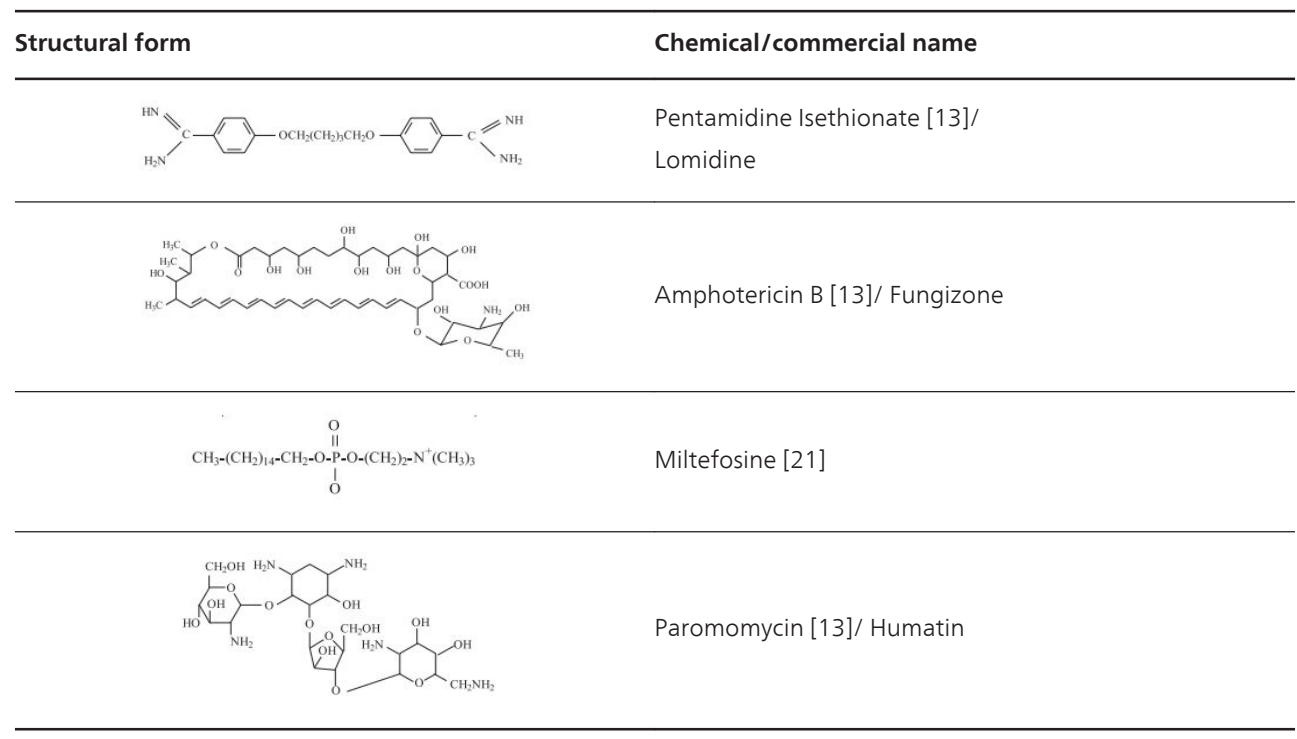

Table 3. Other chemicals used in leishmaniasis therapy, with their respective chemical and commercial names.

\section{Alternative treatments}

Association of some drugs used in leishmaniasis treatment within lipid vesicles called liposomes is one of the alternatives used to reduce the undesirable effects. This association increases the efficiency and the concentrations of these drugs in the tissues, reducing drastically their toxicity $[22,23]$. The mechanism that could explain the effectiveness of liposomes is that they inhibit oxygen consumption by the parasite membrane [24].

One practical exemple of this association happens with Amphotericin B, a compound used for the treatment of calazar patients that are resistant to pentamidine. Amphotericin B can be toxic because they can associate with human cell cholesterol as they do to the ergosterol in the parasite plasmatic membrane [6]. To reduce this inappropriate recognition, there are some commercial formulations that associate the Amphotericin B to lipids (Ambisome, Abelcet, Amphotec). Besides their effectiveness in reducing this drug toxicity, they are very expensive and their use becomes basically impractical in poor countries [25].

However, there are some studies searching some low-cost methods of producing these drugs. One example is a formulation with a similar lipid composition to Abelcet, but with some different variables, such as conformation and molecular weight, that may influence the drug release and action in the organism [26]. This formulation was found to be much more effective and less toxic than Abelcet and, although it is less efficient than Ambisome, it is also less expensive for use in leishmaniasis treatment. 


\section{Vaccines against Leishmaniasis}

Studies for the achievement of a vaccine for leishmaniasis immunization are accomplished since 1940 [27]. In the firsts vaccination process against this disease, scientists didn't know that they were dealing with a microbian ill. This vaccine method was based in the injection of the active wound secretion from one patient to another that was not ill.

From this vaccine, other techniques were developed aiming the raise of the prophylactic efficiency and to avoid some troubles caused by the emergence of a wound. Thus, specialized literature reports four types of vaccines against different types of leishmaniasis: the vaccine based on dead parasites, the vaccine based on living but attenuated parasites, the vaccine with antigenic fragments, and the genic vaccine based on DNA [28].

In a few countries, some clinical trials were already performed but none of the vaccines showed a level of efficiency higher than $80 \%$, a fact that makes unfeasible human tests. This lack of security may be associated to the differentiation between Leishmania species [29], and this differentiation hampers the accomplishment of a wide immunization field.

New investments to the manufacture of a vaccine against leishmaniasis depend on an active biomass of these protozoa. This way, when in contact with the target it may generate an immunological response without generating the disease. But nowadays, the methods for obtaining this biomass $[30,31]$ are very expensive with low profitability, since it generates a very small sample with an inefficient outcome.

\section{New treatment proposals}

Due to intense collateral effects, lots of people who are infected with leishmaniases refuse treatment, and there is the need to new alternative treatments. One of the new possibilities in study nowadays uses natural rubber (NR) membranes with metallic nanoparticles (MNPs) such as silver and gold (SNPs and GNPs, respectively). NR membranes are used as an active support to the MNPs reduction process using precursor salts to obtain metallic particles, and that means that the NR membrane works as a reducing agent as well as a stabilizer for MNPs.

When a promastigote population of Leishmania braziliensis is in the presence of pure natural rubber membranes, there is a significant increase in the log phase of the cells, constituted by a large number of cells at the division process. In the 27th hour after inoculation, the parasite colony remains stable, without large variations in the population cells. In this phase, called stationary phase, the promastigote cells start morphophysiological transformations known as metacyclogenesis. With the end of the stationary phase, an environmental saturation happens with cell waste and lack of nutrients, resulting in the death of cells, phase known as fall.

This decrease could be explained by the natural dynamics of promastigotes in the culture medium. It is expected that after 150 hours, this decrease in the population number occurs because of nutritional deficit and saturation of the cellular environment with cellular excreta 
generated during the period of growth log and stationary phase, but the presence of nanoparticles anticipates the drop in the number of the parasites, probably because these metal components stop the absorption of nutrients of the environment through permeases and transplasmalema transport channels.

However, when promastigotes interact with natural rubber membranes impregnated with silver nanoparticles, a stretch of the first metabolic phase is seen, only with a non-significant growth. In figure 5 is possible to see that the decrease in L. braziliensis is proportional to the increase of silver concentrations in the NR membrane.

Silver nanoparticles toxicity is well known, and although death of the promastigotes may occur, it is believed that changes in the physiological maturation process, such as drastic changes in the surface glycoproteins, happen to the microorganism cell. In that way, the NR membranes with silver nanoparticles when put in the protozoa medium is capable of separating the cells without killing the whole population, eliminating only the ones which did not finalized their metacyclogenesis and are highly infective to the hosts (Figure 6). Immature cells that are not able to generate a disease are kept intact, and this process is what generates an important biomass to a future study focus a vaccine against this disease.

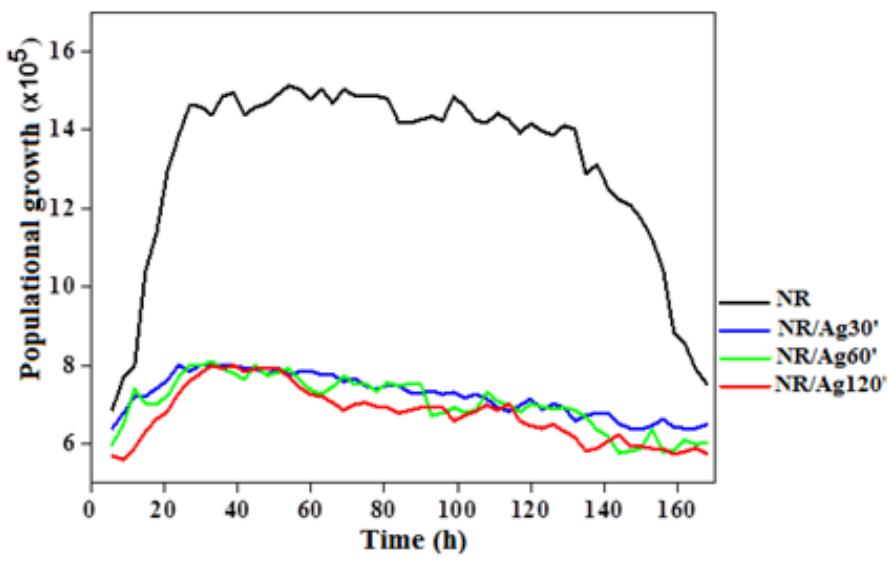

Figure 5. Populational growth of Leishmania braziliensis promastigotes in contact with natural rubber membranes and natural rubber membranes impregnated with silver nanoparticles using in situ reduction.

Natural rubber membranes containing GNPs (NR/Au) also decrease the population growth rate, showing a lower index of living promastigotes (attached to the membrane surface) depending on the amount of nanoparticles deposited in the membrane surface. Formation and growth of the GNPs within the NR membranes, by reduction of $\mathrm{Au}^{3+}$, was monitored following the Plasmon absorption band. Reducing agents for GNPs formation are likely to be the carboxylic functional groups present in the NR. Nanoparticle, which produces an increase of the intensity of the distinctive plasmon absorption band of GNPs centered at approximately $560 \mathrm{~nm}$, as shown in Figure 7 [33]. 

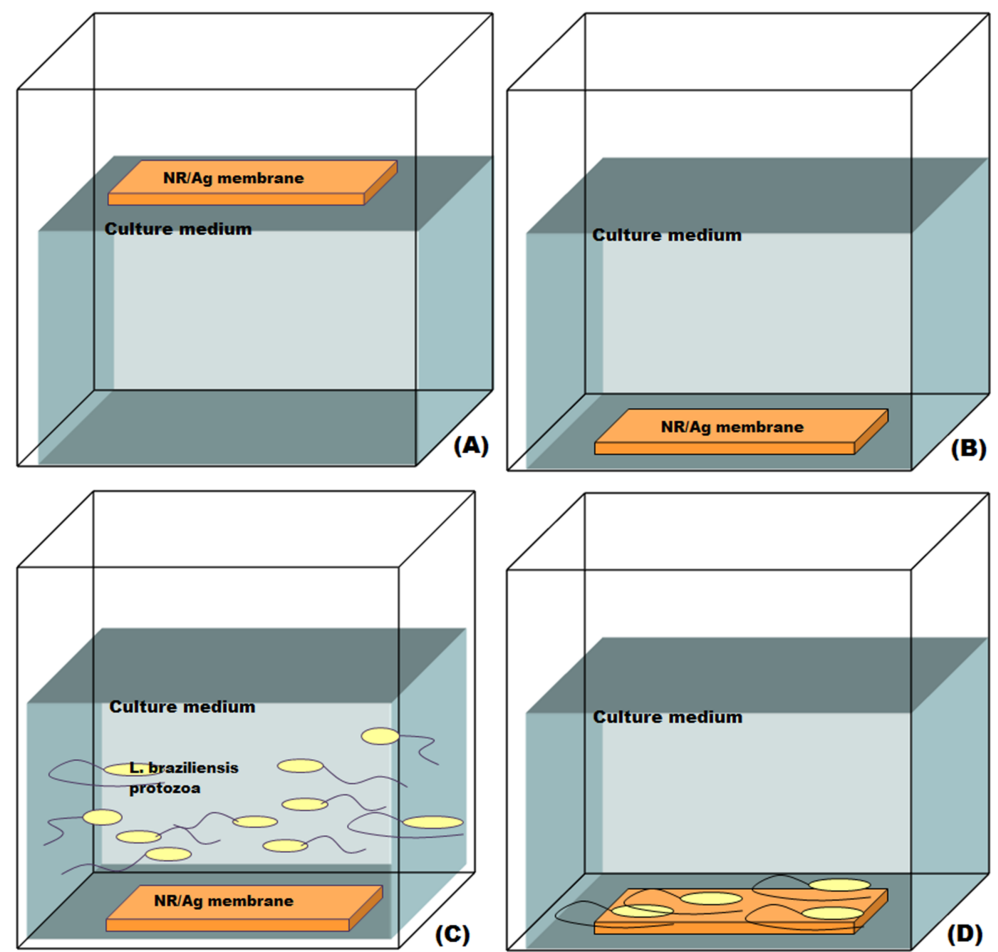

Figure 6. Separation Process of Leishmania in contact with NR membranes with silver (Ag) nanoparticles; (a) and (b) NR/Ag membranas in culture medium; (c) Leishmania braziliensis in contact to NR/Ag membranes; (d) Separated protozoa attached to NR/Ag membranes.

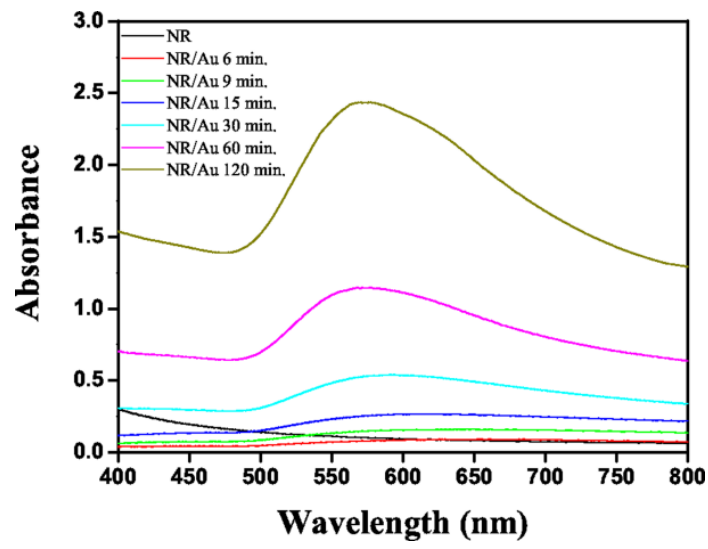

Figure 7. UV-Vis spectroscopy of natural rubber/gold nanoparticles prepared at different gold reduction times, compared to raw natural rubber membrane (preparation of the membrane annealed at $65^{\circ} \mathrm{C}$ ). 
Analyses by scanning electron microscopy (ESEM) were performed for natural rubber membranes with gold nanoparticles, obtained at a reduction time of $30 \mathrm{~min}$ (Figure 8). Apparently, there is a homogenous distribution of nanoparticle occupying the entire surface of the membrane, but by amplifying the images, one can observe the formation of many nanoparticles in sites, and among them, nanoparticles dispersed on the surface of the polymeric matrix. In Figure 8 (d) the small spherical nanoparticles can be seen agglomerated over reduction sites, forming aggregates at an exorbitant amount. Nanoparticles sizes were measured and are very similar, with an average of approximately $48 \mathrm{~nm}$. These results are in agreement with the analysis by UV-Vis spectroscopy.
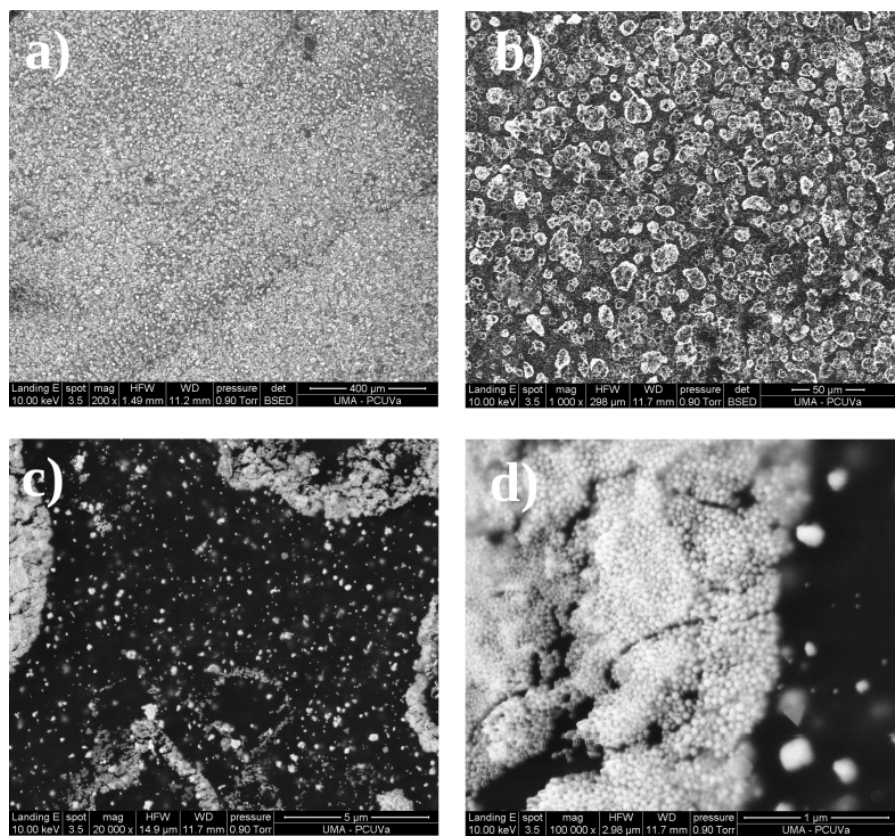

Figure 8. SEM analysis of NR/Au membrane obtained at 30 minutes of reduction. Magnification of (a) 200 times; (b) 1,000 times; (c) 20,000 times; (d) 100,000 times.

Natural rubber membranes, with colloidal incorporation of gold nanoparticles, were synthesized by in situ method (NR/Au) at different reduction times, in order to evaluate the physiological behavior of L. braziliensis promastigotes cultured in axenic medium, in a colony of seven weeks old, evaluating the increase kinetics in relation to the permanence time of the membranes in the culture medium as a function of reduction time and temperature of preparation of natural rubber membranes.

It was observed that the inclusion of NR/Au membrane in culture generates a delayed onset of growth in culture until about 24 hours, with a reduction in the development population proportional to the time reduction of the gold nanoparticles. It is also noted that the NR/Au 
$120 \mathrm{~min}$. membrane presents the best results with regard to growth inhibition of promastigote population in culture medium. NR/Au $30 \mathrm{~min}$ membrane has a sharper decline in curve kinetics attributable to death of promastigotes from 120 hours. Upon analysis completion of growth medium, the membranes prepared for times of 30, 60 and 120 minutes showed similar results, with the number of promastigotes in solution smaller than inserted into the initial population.

According to the results the best allowed reduction time is 120 minutes, due to its greater population growth inhibition, and was then assessed the influence of the thermal treatment by the casting preparation method of membrane natural rubber annealed at 60,80 and $120^{\circ} \mathrm{C}$, and then used as substrates for the reduction of nanoparticles. Results obtained for the kinetics of increase in solution are shown in Figure 9.

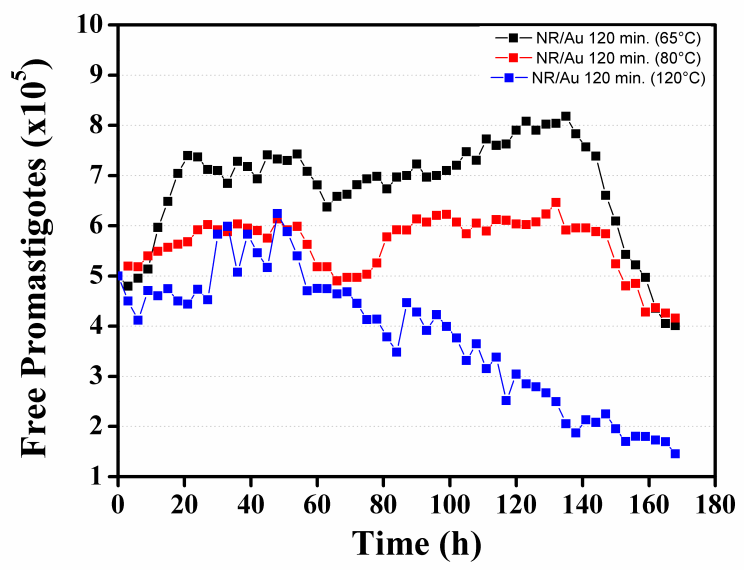

Figure 9. Analysis of the kinetics of increase of promastigotes in solution in the presence of natural rubber membranes prepared by the casting method, at temperatures of 65,80 and $120^{\circ} \mathrm{C}$, and then incorporated gold nanoparticles to the time reduction of 120 minutes.

It is observed that with increasing of thermal treatment temperature in the preparation of NR membranes the population growth factor in culture decreases, reaching scores lower than $2 \times 10^{5}$ promastigotes (less than half of the inoculum) to membranes prepared at $120{ }^{\circ} \mathrm{C}$ at a reduced nanoparticles to 120 minutes. It is known from promastigotes reactivity with carboxylic and nitrogenated groups, the same reactive groups and assigned to the reduction of nanoparticles. Higher the temperature of thermal treatment used in the preparation of NR membranes, smaller the amount of nanoparticles embedded in the membrane surface, attributed to the fact that there is further degradation of the active components of the membrane with increasing temperature. Thus, it is attributed the decrease in population growth in solution to generate more reactive nanoparticles or selective proteins contained in the culture medium, inhibiting the development of promastigotes, and may also be linked to decreasing 
of active cores on the membrane surface, or even liberation of radicals in the culture medium attributed to membrane preparation temperature, but the level of promastigotes in culture medium still remains considerable, so that in an attempt to develop a smart skin bandage incorporated into methylene blue molecules on Au particles to photodynamic treatment, due to the drug photosensitivity.

Membranes of pure natural rubber and with incorporation of gold nanoparticles and methylene blue were inserted in the culture medium and the behavior of L. braziliensis promastigotes were evaluated through the population growth kinetics and morphophysiological analysis. Results regarding to population growth obtained for protozoan eluted to membranes prepared at $120^{\circ} \mathrm{C}$ of thermal treatment are presented in Figure 10.

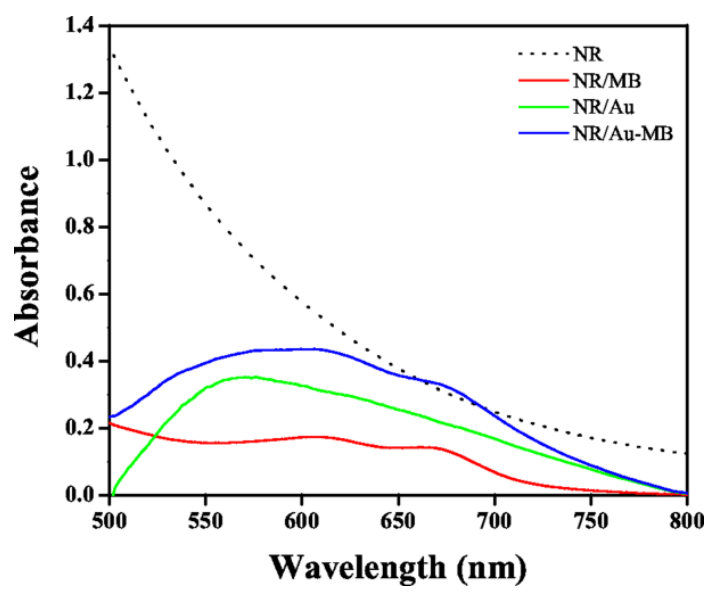

Figure 10. Molecular spectroscopy in UV-Vis region of natural rubber membranes with gold nanoparticles for reduction time of $120 \mathrm{~min}$ and functionalization with methylene blue molecules, compared to pure natural rubber membranes.

To improve the studies, blue methylene was incorporated to the NR membrane. This incorporation does not show toxic interaction with promastigotes, since the population growth usually develops when inserted NR/MB membranes. Moreover, when irradiated culture media containing BM molecules, a deficit in the population growth is observed. For NR/MB membranes irradiated with blue light total elimination of promastigotes is observed in about 120 hours, whereas when MB molecules deposited on gold nanoparticles this time decreases to about 60 hours. This fact is attributed to the amplification effect of surface commonly evaluated by micro-Raman spectroscopy, so that the metallic particles act as sites for amplification of energy absorbed by the anchored molecules.

The electrons assume an excited energy state, and during the release of the absorbed energy active radicals are generated, with interaction with the environment where are MB molecules. In the first instance, electrons from MB molecules, when returning to the ground state emit enough energy to excite the oxygen present in the medium converting to superoxides or 
peroxides radials. This transfer may occur also by interaction with hydrogen atoms present in other molecules to form radicals, e.g. hydroxyl, also with potential toxic to cells, or as in this case, the promastigotes. If the emission energy is derived from a transition to a triplet state of the photosensitizer, one can obtain the formation of a singlet oxygen molecule and an oxidizing agent more toxic to cells [34-38].

\section{Conclusion}

Membranes with metal nanoparticles significantly influences the development of the protozoan L. braziliensis, thus they can be used for different applications.

Membranes with SNPs can separate the cells at different stages of maturation. In this case, we obtain a biomass feasible to manufacture a vaccine for the purpose of immunization against leishmaniasis.

Membranes with GNPs can used for a possible treatment of cutaneous leishmaniasis, once the cell death is cause due to interaction between the gold and protozoan. NR/MB membranes can be direct to the photosensitive treatment of lesions and so accelerate the closing of the same. Thus, membranes with metallic nanoparticles are a viable and the low cost for both the immunization procedure and in the treatment process because the disease is already established. In addition, treatment with the application of membranes with MNPs dramatically reduces the side effects caused by conventional treatment.

\section{Author details}

Aldo Eloizo Job*, Alexandre Fioravante de Siqueira, Caroline Silva Danna, Felipe Silva Bellucci, Flávio Camargo Cabrera, Leandra Ernst Kerche Silva and Pedro Henrique Benites Aoki

*Address all correspondence to: job@fct.unesp.br

Department of Physics, Chemistry and Biology, Univ Estadual Paulista, Presidente Prudente, Sao Paulo, Brazil

\section{References}

[1] Tato Lmp Et Al. Colloids And Surfaces B: Biointerfaces. 2009;68: 55-60.

[2] Ministério Da Saúde, Secretaria De Vigilância Em Saúde. Manual De Vigilância Da Leishmaniose Tegumentar Americana. Brasília: Editora Do Ministério Da Saúde; 2007. 
[3] Goto H, Lindoso Jal. Expert Review Of Anti-Infective Therapy. 2010; 8(4): 419-433.

[4] Späth Gf Et Al. Proceedings Of The National Academy Of Sciences (Usa). 2000; 97: 9258-9263.

[5] Rath S Et Al. Química Nova. 2003;26(4): 550-555.

[6] Santos Od. Parasitology Research. 2008;103: 1-10.

[7] Grimaldi, G.; Tesh, A. B.; Mcmahon, P. D. The American Journal Of Tropical Medicine And Hygiene, 41, 689, 1989.

[8] Http://Www.Dpd.Cdc.Gov/Dpdx/Html/Leishmaniasis.Htm

[9] Vianna, G. O. Anais Do $7^{\circ}$ Congresso Brasileiro De Medicina E Cirurgia, 4, 426, 1912.

[10] Berman, J. D. Reviews Of Infectious Disease, 10, 560, 1988.

[11] Marsden, P. D. Revista Da Sociedade Brasileira De Medicina Tropical, 18, 187, 1985.

[12] Balaña-Fouce, R.; Reguera, R. M.; Cubría, C.; Ordóñez, D. General Pharmacology, 30, 435, 1998.

[13] Sereno, D.; Lemesre, J. L. Antimicrobial Agents And Chemotherapy, 41, 972, 1997.

[14] Berman, J. D.; Wyler, D. J. The Journal Of Infectious Diseases, 142, 83,1980.

[15] Limongi, J. P. In Farmacodinâmica; Corbett, C. E., Ed. Livraria Editora Artes Médicas, São Paulo, 1973, Cap. 61.

[16] D’Rea, J. G. Et Al. Clinical Chemistry, 33, 2081, 1987.

[17] Roberts, W. L.; Mcmurray, W. J.; Rainey, P. M. Antimicrobial Agents And Chemotherapy, 42, 1076, 1998.

[18] Miekeley, N.; Mortari, S. R.; Schubach, A. O. Analytical And Bioanalytical Chemistry, 372, 495, 2002.

[19] Doua, F. Et Al. American Journal Of Tropical Medicine And Hygiene, 55, 586, 1996.

[20] Rang, H. P.; Dale, M. M.; In Pharmacology, 2nd Edition, Churchill Livingstone: Uk, 1991.

[21] Fischer, C.; Voss, A.; Engel, J. Medical Microbiology And Immunology, 190, 85, 2001.

[22] Desjeux, P; Alvar, J. Annals Of Tropical Medicine And Parasitology, 97 (1), 3-15, 2003.

[23] Gontijo, B.; Carvalho, M. L. R. Revista De Sociedade Brasileira De Medicina Tropical, 36 (1), 71-80, 2003.

[24] Rosa, M. S. S. Et Al. Antimicrobial Agents And Chemotherapy, 47 (6), 1895-1901, 2003. 
[25] Golenser, J. Et Al. Antimicrobial Agents And Chemotherapy, 43 (9), 2209-2214, 1999.

[26] Larabi, M. Et Al. Antimicrobial Agents And Chemotherapy, 47 (12), 3774-3779, 2003.

[27] Grimaldi Jr.,G. Memórias Do Instituto Oswaldo Cruz, 90, 553- 556, 1995.

[28] Handman, E. Parasitology Today, 13, 236-237, 1997.

[29] Antunes, C. M. F. Et Al. International Journal Of Epidemiology, 15, 572-579, 1986.

[30] Dórea, R. C. C., Et Al. Brazilian Journal Of Microbiology, 34, 1, 7-20, 2003.

[31] Kohri, M. Et Al, European Polymer Journal, 47, 2351-60, 2011.

[32] Barbosa-Filho, C.G. Et Al. Experimental Parasitology, 130, 152-158, 2012.

[33] Aroca, R. F.; Alvarez-Puebla, R. A.; Pieczonka, N.; Sanchez-Cortez, S.; Garcia-Ramos, J. V. Adv. Colloid Interface Sci., 116 (1-3), 45-61, 2005.

[34] Peloi, L. S. Et Al. Exp. Parasitol., 128 (4), 353, 2011.

[35] Prates, R. A. Et Al. Journal Of Photochemistry And Photobiology, 86 (1), 70-76, 2007.

[36] Wainwright, M. Journal Of Antimicrobial Chemotherapy, 42, 13-28, 1998.

[37] Miyabe, M. Braz. Oral Res., 25 (3), 230, 2011.

[38] Longo, J. P. F.; Azevedo, R. B. J. Dent. Clin. Res., 6 (3), 249-257, 2010. 
